and since $B\left(x_{2}, y_{2}\right)$ lies on (3)

$$
a_{1} x_{2}+b_{1} y_{2}+c_{1}=+k c_{2} \text {. }
$$

Adding, and dividing by 2,

$$
a_{1} \frac{x_{1}+x_{2}}{2}+b_{1} \frac{y_{1}+y_{2}}{2}+c_{1}=0 .
$$

Therefore the mid-point of $A B$ lies on (1).

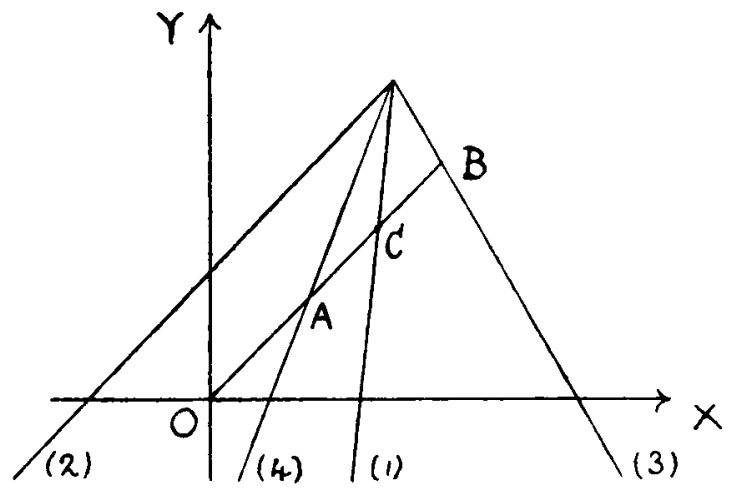

N. M'Arthur.

\title{
Trigonometrical Ratios of the half-angles of a Triangle (Geometrical Proofs).
}

1. $A B C$ is a triangle; bisect angle $A$ by $A E$; produce $A B$; draw $B D F$ and $C E G$ perpendicular to $A E$; join $F G$.

$G C F B$ is a cyclic trapezium

$$
\begin{aligned}
& \therefore G C . F B+C F . B G=B C . F G \text {, } \\
& \therefore \quad 2 E C .2 D B+(b-c)^{2}=a^{2} \text {, } \\
& \therefore \quad 4 E C, D B=a^{2}-(b-c)^{2} \\
& =(a-b+c)(a+b-c) \\
& =4(s-b)(s-c) \text {, } \\
& \therefore \quad E C, D B=(s-b)(s-c) \text {. }
\end{aligned}
$$




$$
\begin{aligned}
& \text { Now } \sin \frac{A}{2}=\frac{E C}{A C}=\frac{D B}{A B}, \\
& \therefore \quad \sin ^{2} \frac{A}{2}=\frac{E C \cdot D B}{A C \cdot A B}=\frac{(8-b)(8-c)}{b c}, \\
& \therefore \quad \sin \frac{A}{2}=\sqrt{\frac{(s-b)(s-c)}{b c}} .
\end{aligned}
$$

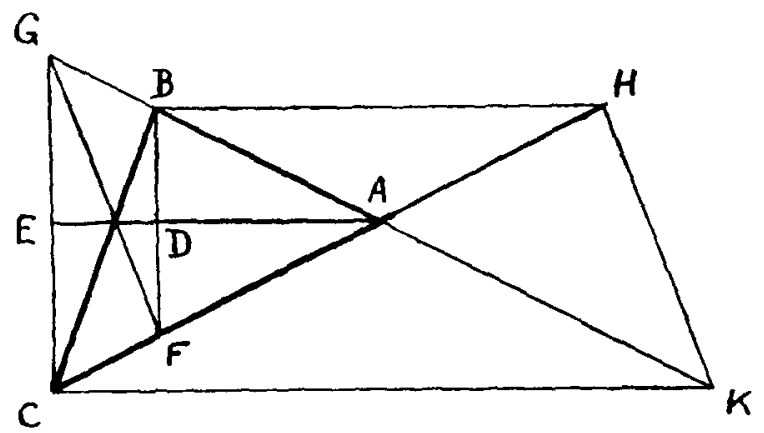

2. Produce $C A$ to $H$ making $A H=A B$; produce $B A$ to $K$ making $A K=A C$; join $H K, B H, C K$.

$C K H B$ is a cyclic trapezium

$$
\begin{aligned}
& \therefore \quad C K \cdot B H+B C \cdot H K=B K . H C \text {, } \\
& \therefore \quad 2 A E .2 A D+a^{2}=(b+c)^{2} \text {, } \\
& \therefore \quad 4 A E \cdot A D=(b+c)^{2}-a^{2} \\
& =(b+c+a)(b+c-a) \\
& =48(s-a) \text {, } \\
& \therefore \quad A E \cdot A D=8(8-a) \text {. }
\end{aligned}
$$

Now $\cos \frac{A}{2}=\frac{A E}{A C}=\frac{A D}{A B}$,

$$
\begin{aligned}
& \therefore \quad \cos ^{2} \frac{A}{2}=\frac{A E \cdot A D}{A C \cdot A B}=\frac{8(8-a)}{b c}, \\
& \therefore \quad \cos \frac{A}{2}=\sqrt{\frac{8(8-a)}{b c}} .
\end{aligned}
$$


3. Again $\tan \frac{A}{2}=\frac{E C}{A E}=\frac{D B}{A D}$,

$$
\begin{aligned}
& \therefore \quad \tan ^{2} \frac{A}{2}=\frac{E C \cdot D B}{A E \cdot A D}=\frac{(s-b)(s-c)}{s(s-a)}, \\
& \therefore \quad \tan \frac{A}{2}=\sqrt{\frac{(s-b)(s-c)}{s(s-a)}} .
\end{aligned}
$$

4. The formula of the area of a triangle in terms of the sides may be obtained by the direct use of these ratios, thus

$$
\begin{aligned}
\triangle A B C & =\triangle B C K-\triangle A C K \\
& =\frac{1}{2} C K(C E+D B)-\frac{1}{2} C K \cdot C E \\
& =\frac{1}{2} C K \cdot D B \\
& =A E \cdot D B \\
& =b \cos \frac{A}{2} \cdot c \sin \frac{A}{2} \\
& =b c \sqrt{\frac{s(s-a)}{b c}} \sqrt{\frac{(s-b)(s-c)}{b c}} \\
& =\sqrt{8(s-a)(s-b)(s-c)} .
\end{aligned}
$$

Alex. D. Russill.

Note re Prior Publication.

We are in receipt of the following communication from Mr A. D. Russell, dated 20/12/20:-

"I have a p.c. from $M_{r}$ R. C. Archibald, Editor of the American Math. Monthly, in which he points out that my "Proof of the Law of Tangents," Proc. Edin. Math. Soc., Vol. XXXVIII., p. 58 (Nov. 1920), is identical with that by Mr Cheney published in the Amer. Math. Monthly, Feb. 1920. I need scarcely say that the publication of $\mathrm{Mr}$ Cheney's proof was quite unknown to me, but at the same time I should like to state that I have used the proof in my classes for a few years now. I find, for example, from some former pupils that their notebooks fully establish the fact that I gave the proof to a class in Falkirk Science and Art School on 6th Feb. 1918." 